\title{
Eggerthella lenta Bacteremia in a Middle-Aged Healthy Man with Acute Hepatic Abscess: Case Report and Literature Review, 1970-2020
}

\author{
Jiazheng Wang ${ }^{1, *}$ \\ Rui Guo',* \\ Wanshan $\mathrm{Ma}^{\mathrm{I}}$ \\ Xiutao Dong' \\ Shaofeng Yan $^{2}$ \\ Wenyan Xie
}

'Department of Clinical Laboratory Medicine, The First Affiliated Hospital of Shandong First Medical University \& Shandong Provincial Qianfoshan Hospital, Shandong Medicine and Health Key Laboratory of Laboratory Medicine, Jinan, People's Republic of China; ${ }^{2}$ Department of Neurosurgery, The First Affiliated Hospital of Shandong First Medical University \& Shandong Provincial Qianfoshan Hospital, Shandong Medicine and Health Key Laboratory of

Neurosurgery, Jinan, People's Republic of China

*These authors contributed equally to this work

Correspondence: Wenyan Xie Department of Clinical Laboratory Medicine, The First Affiliated Hospital of Shandong First Medical University \& Shandong Provincial Qianfoshan Hospital, Shandong Medicine and Health Key Laboratory of Laboratory Medicine, 16766 Jingshi Road, Jinan, Shandong Province, People's Republic of China Tel +86 I5I69|7876I

Email cunnuanhuakaisd@I63.com

Shaofeng Yan

Department of Neurosurgery, The First Affiliated Hospital of Shandong First Medical University \& Shandong Provincial Qianfoshan Hospital, Shandong Medicine and Health Key Laboratory of Laboratory Medicine, 16766 Jingshi Road, Jinan, Shandong Province, People's Republic of China

Tel +86 I5662793799

Email sdqyyan@163.com

\begin{abstract}
Eggerthella lenta (E. lenta) is a rare but significant human emerging pathogen. Infections caused by it are rare and little-known, both on clinical and therapeutical aspects, in spite of new emergence of bacteria isolation and identification techniques. In this article, we report a case involving a previously healthy 52-year-old man suffering from a newly diagnosed hepatic abscess who developed E. lenta bacteremia, which was treated successfully using empirical therapy with ertapenem and teicoplanin. To the best of our knowledge, this is the first documented report of $E$. lenta bacteremia related specifically to liver abscess. Cases related to this bacterial species are infrequent and sporadic; thus, we reviewed English literature on E. lenta infection in PubMed/MEDLINE in the last 50 years. A total of 31 sporadic cases were identified. The majority of patients were male (71\%), had an average age of 54.3 years and presented predisposing conditions, such as digestive system trouble (45.2\%), immunocompromised state $(25.8 \%)$ or risk factors (22.6\%). Two of the cases had more than one predisposing factors. Fever was common (93.5\%). Average days to diagnosis of them were 6.8 days. MALDI-TOF MS is emerging as a fast and useful tool in the identification of it. Teicoplanin, vancomycin, amoxicillin-clavulanate, metronidazole, clindamycin, cefoxitin, chloramphenicol, and carbapenems appear to be the most used antibiotic treatment options. The purpose of this review is to increase awareness about the clinical infections caused by $E$. lenta.
\end{abstract}

Keywords: Eggerthella lenta, infections, antibiotic therapy, diagnosis, risk factors

\section{Introduction}

Eggerthella lenta (E. lenta) is an obligate anaerobic, non-motile, non-spore-forming Gram-positive bacillus found in the indigenous intestinal microflora of human. ${ }^{1}$ It can grow as a single organism, in pairs or in short chains. This organism was initially isolated from human faeces and firstly identified by Arnold H. Eggerth in 1935. ${ }^{1}$ Its original name Eubacterium lentum was changed to E. lenta on the basis of 16S ribosomal RNA gene sequencing in 1999, and concurrently transferred to its own distinct genus. ${ }^{2,3}$ Its complete genomic sequence was first described in $2009{ }^{4}$

$E$. lenta has been identified as the causative pathogen of bacteremia, sinusitis, appendicitis, decubitus ulcers, spondylodiscitis, splenic abscesses and pelvic abscesses. ${ }^{5,6}$ The clinical spectrum of the diseases caused by this organism, as well as the risk factors, laboratory examination, diagnosis and antibiotic treatment has not been well described systematically so far, and the cases of $E$. lenta infection in published literature are scarce. This scarcity may be because the identification of 
anaerobic bacteria is difficult, expensive, and timeconsuming, and these bacteria are therefore historically less frequently recognized. Herein, we present a case of E. lenta bacteremia in a previously healthy middle-aged male suffering from liver abscess, and conduct a retrospective analysis of 30 sporadic cases of $E$. lenta infection that were reported in the last 50 years.

\section{Case Presentation}

A 52-year-old Chinese male was admitted to our hospital with complaints of right upper quadrant pain and discomfort for one week. His symptoms began approximately one week before admission. His right upper quadrant pain had no obvious predisposing factors. The pain was paroxysmal, accompanied by abdominal fullness and distention and worsened after meals. The patient denied any obvious nausea, vomiting, stiffness, fever and other symptoms. He had no past medical history and had no history of close contact with infected individuals, recent travel or any abdominal trauma. He sought care in a local hospital where he was diagnosed with hepatic abscess and treated with hepatic puncture and drainage 1 day earlier.

The patient and family requested a transfer to our hospital for further treatment. He was afebrile on the day of admission and had a pulse rate of 80 beats/minute, a blood pressure of $118 / 73 \mathrm{mmHg}$ and a respiratory rate of 26 breaths/minute. Laboratory investigation showed a leukocyte count of $16.4 \times 10^{9} / \mathrm{L}$ (neutrophil $94.5 \%$ ), a haemoglobin level of $85 \mathrm{~g} / \mathrm{L}$, a platelet count of $240 \times 10^{9} / \mathrm{L}$, a procalcitonin (PCT) level of $2.3 \mathrm{ng} / \mathrm{mL}$ and a plasma fibrinogen level of $7.48 \mathrm{~g} / \mathrm{L}$. His liver and renal function tests were slightly abnormal; he had a serum glutamic oxalacetic transaminase level of $67 \mathrm{U} / \mathrm{L}$, a glutamic-pyruvic transaminase level of $83 \mathrm{U} / \mathrm{L}$, a cholinesterase level of $1360 \mathrm{U} / \mathrm{L}$, total protein and albumin levels of 50.8 and $21.5 \mathrm{~g} / \mathrm{L}$, a blood urea nitrogen level of $10.8 \mathrm{mmol} / \mathrm{L}$ and a creatinine level of $51.3 \mathrm{umol} /$ L. He was negative for HIV and syphilis. However, he had a complaint of obvious abdominal distension with mild bellyache and extreme thirst on day 2, right abdominal tenderness without rebound or guarding on exam and a temperature of $36.8^{\circ} \mathrm{C}$. The drainage catheter yield of $150 \mathrm{~mL}$ of fulvous fluid was submitted to the microbiology laboratory for analysis. Abdominal and pelvic computed tomography (CT) scan was immediately performed to evaluate the change in his condition. The CT image (Figure 1 and Figure 1 supplement) shows an irregular, slightly low-density lesion in the right posterior hepatic

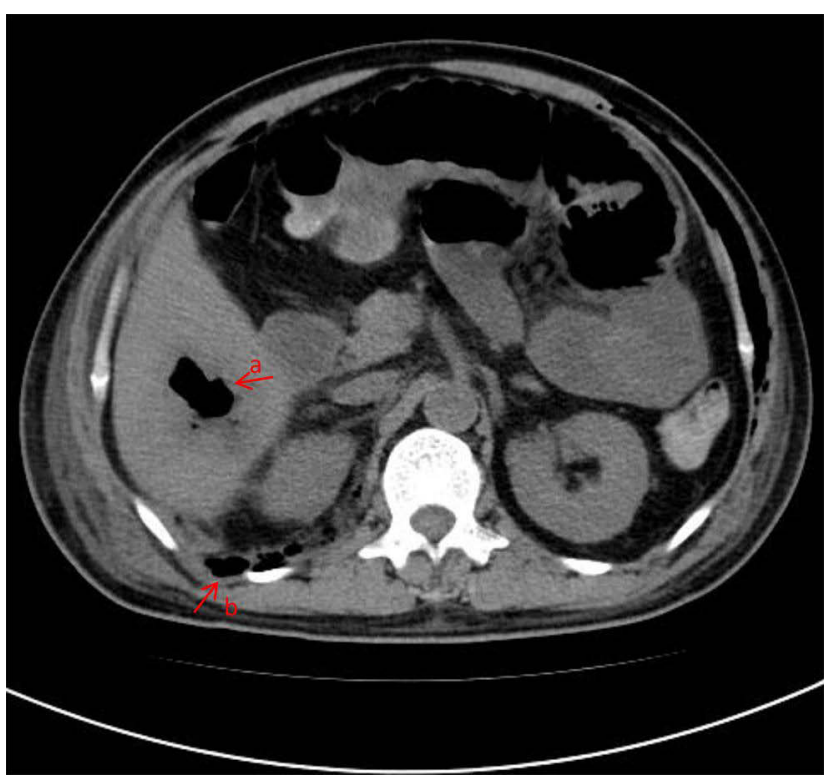

Figure I CT image showing liver abscess, multiple gas accumulation and dilation in the bowel, and retroperitoneal infection. a. Liver abscess; b. Retroperitoneal infection.

lobe with a size of $41 \mathrm{~mm} \times 56 \mathrm{~mm}$ with an obscured boundary and a gas density shadow inside. A liquid density shadow and high-density drainage tube shadow are present around the lower margin of liver. A round-like low-density lesion of about $0.8 \mathrm{~cm}$ in diameter is located in the right lobe of the liver. The appendix thickened to about $20 \mathrm{~mm}$ in diameter, and the structure of the ascending colon near the ileocecal region became disorganised. Multiple gas accumulation and dilation in the bowel and air-fluid levels inside the abdomen, which likely represent hepatic abscesses, ileus, mild ascites, appendicitis, liver cyst, abdominal infection and peritonitis, were observed.

The patient presented clinical symptoms of pre-shock, such as insufficient blood pressure of 93/59 $\mathrm{mmHg}$, and was taken to the operating room for urgent exploratory laparotomy. Intraoperative examination revealed the presence of fulvous purulent exudate and necrotic tissue filled the extraperitoneal space and abdominal cavity, partial postnecrotic defect in the peritoneum and massive epiploon adhesion in the right upper abdomen. Two perforations were found after the separation of adhesion. One is located under the right side of the liver with a diameter approximately of $15 \mathrm{~mm}$, and the other is located in the right ascending colon about $100 \mathrm{~mm}$ from the ileocecal region. The patient had ileocecal resection; partial resection of the ascending colon; ileostomy and drainage of hepatic, abdominal and extraperitoneal abscesses. 
His postoperative course was eventful with orotracheal intubation, hypotension, anemia, fever and transferred to the intensive care unit (ICU) for better monitoring. Noradrenaline was pumped to maintain his blood pressure, ventilator was used to assist breathing, and intravenous hydration was used to enlarge blood capacity. Moreover, nutritional support therapy and blood transfusion were also used. Blood cultures and drainage fluids were sent and he was empirically started on intravenous tigecycline and piperacillin/tazobactam (4.5g, tid, iv.drip) for broader coverage while awaiting the culture results. Body temperature, routine blood count, procalcitonin level and C-reactive protein (CRP) level were closely monitored dynamically.

On Day 2 after surgery, his temperature went up to $39.3^{\circ} \mathrm{C}$. The electrocardiogram monitor showed a pulse rate of 130 beats/minute. Haematological values revealed an elevated leukocyte count of $35.9 \times 10^{9}$ cells/L (neutrophils: $92.4 \%$ ), an upward PCT level of $8.15 \mathrm{ng} / \mathrm{mL}$ and an raised CRP level of $174 \mathrm{mg} / \mathrm{L}$. Blood samples were taken for aerobic and anaerobic cultures again, and the previous empiric antibiotic regimen was still continued. Three days later, the two sets of anaerobic blood culture vials drawn postoperatively were reported to be positive. Gram stain revealed short Gram-positive bacillus without spores. The positive culture broth was inoculated onto blood agar base medium and anaerobically incubated for three more days. A colony from the agar plate was identified as E. lenta by matrix-assisted laser desorption/ionization time-of-flight mass spectrometry (MALDI-TOF MS) (Bruker Daltonics, Germany). Antimicrobial susceptibility testing of E. lenta was not performed because of limitations in laboratory conditions.

Although the patient had good clinical response to empirical tigecycline and piperacillin/tazobactam therapy, his plasma fibrinogen fell to $1.0 \mathrm{~g} / \mathrm{L}$ which was presumed to be an adverse reaction to tigecycline. Thus, tigecycline was replaced by teicoplanin. Piperacillin/tazobactam was discontinued and ertapenem was added to teicoplanin to target $E$. lenta bacteremia and maintain broader coverage. Simultaneously, cultures of the drainage fluid were obtained with the isolation of organism as Escherichia coli which also susceptible to ertapenem. The patient was treated with ertapenem and teicoplanin, his fever, leukocytosis, PCT level and CRP level promptly improved. He was transferred to the general ward after 3 days. Subsequently, he was administered ertapenem and teicoplanin for another 14 days in conjunction with debridement, dressing change and symptomatic supportive treatment. Repeated blood cultures after the introduction of antibiotics were negative. Repeat CT revealed that the size of the hepatic abscess and the amount of ascites decreased. After a further 7-day course of intravenous ertapenem and teicoplanin, his symptoms achieved further alleviation, and he was discharged from the hospital with a prescription for oral antibiotics (clindamycin). He remained well as an outpatient without a recurrence of fever, and a complete resolution of the abscess was found at follow-up with a six-week course of intravenous ertapenem.

\section{Literature Review Methods}

We performed an electronic search on PubMed/MEDLINE used the key words "Eggerthella lenta" to systematically search for related case studies in English published from 1970 through 2020. "Eubacterium lentum" was added to the search terms because Eggerthella lenta was previously known as Eubacterium lentum. We also retrieved the references cited in the papers for additional published case reports. Three microbiologists and one clinician cooperated and finished the work, and a database was established. The collected information included general characteristics, outcomes, underlying diseases and/or predisposing factors, laboratory examinations/clinical manifestations, diagnostic test and time until diagnosis as well as AST and antibiotic treatment.

\section{Results}

Our PubMed/MEDLINE search (1970-2020) yielded 30 case reports of sporadic E. lenta infection published in the English language to date. The clinical characteristics and outcomes of the 30 cases, together with our case, are shown in Table 1.

\section{General Characteristics}

The average age was 54.3 years (range 8-94 years), and only $9 / 31(29.0 \%)$ were female. The male-to-female ratio was 2.4:1. The first case was described in 1978, and 25 sporadic cases were reported since 2003. Thirteen cases (13/31) were reported from North America, and 84.6\% $(11 / 13)$ of which came from the United States. Nine cases originated from Asia, of which four (44.4\%) and two $(22.2 \%)$ cases were from China and Pakistan, respectively. Seven cases came from the Europe and two cases from the Oceania, no case was reported in the South America and Africa (Figure 2). Regarding infection 


\begin{tabular}{|c|c|c|c|c|c|c|c|c|c|c|}
\hline$\dot{\bar{\sigma}} \dot{z}$ & $\Xi$ & $\sqrt{2}$ & ฐ & ఫ & $\Xi$ & $\Xi$ & ฐ & $\bar{\Xi}$ & $\bar{\Xi}$ & ฮ \\
\hline 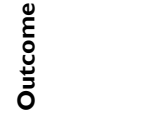 & 总 & 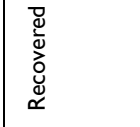 & 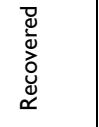 & 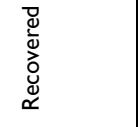 & 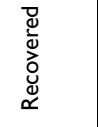 & 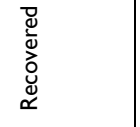 & 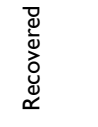 & 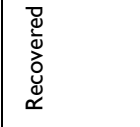 & 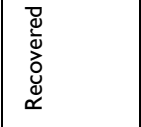 & 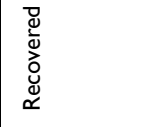 \\
\hline 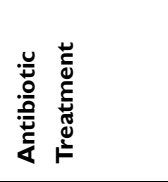 & 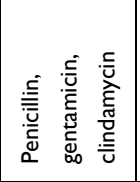 & 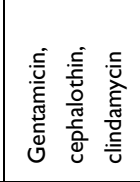 & 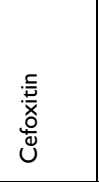 & 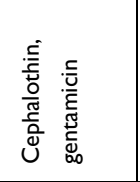 & 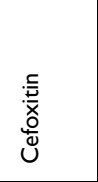 & 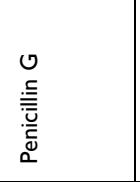 & 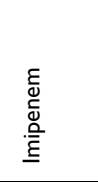 & 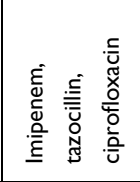 & 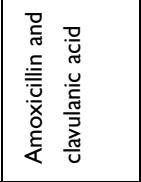 & 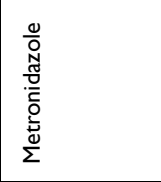 \\
\hline 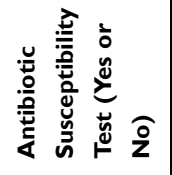 & z & $\stackrel{\circ}{z}$ & $\stackrel{\check{\nu}}{\check{\nu}}$ & 1 & $\stackrel{\tilde{\nu}}{\tau}$ & $\stackrel{\tilde{\nu}}{\check{\nu}}$ & $\stackrel{\tilde{\nu}}{\tau}$ & $\stackrel{\mathscr{\Perp}}{\rightleftharpoons}$ & $\stackrel{\mathscr{\varkappa}}{\sim}$ & $\stackrel{\tilde{\Xi}}{\tau}$ \\
\hline 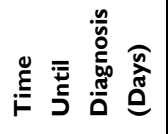 & 1 & I & 1 & 1 & 1 & 1 & 1 & $\begin{array}{l}\infty \\
\text { तิ } \\
\text { वิ }\end{array}$ & $\begin{array}{l}\infty \\
\text { đิ } \\
0\end{array}$ & $\begin{array}{l}\infty \\
\text { O̊ } \\
\text { O̊ }\end{array}$ \\
\hline 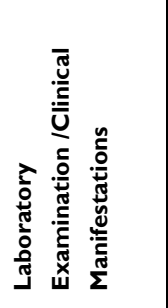 & 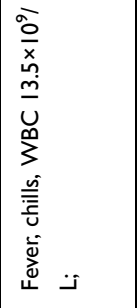 & 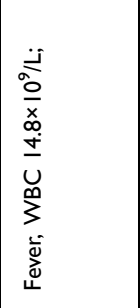 & 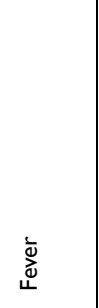 & 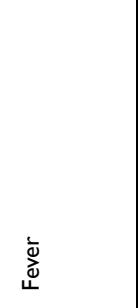 & 㐫 & 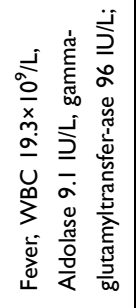 & 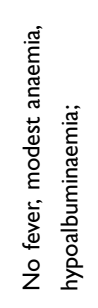 & 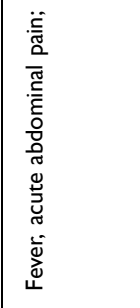 & 产 & 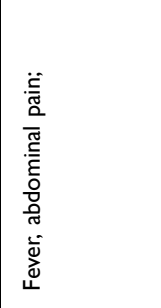 \\
\hline 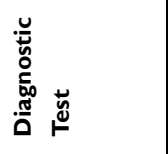 & 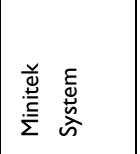 & 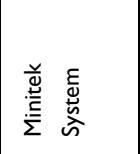 & 1 & 1 & 1 & $\frac{\mathbb{s}}{\stackrel{4}{\alpha}}$ & 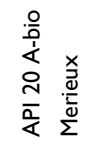 & 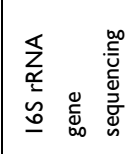 & 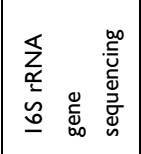 & 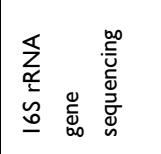 \\
\hline 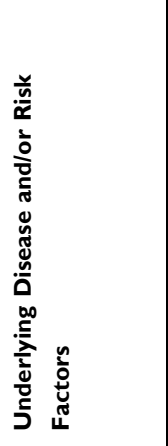 & 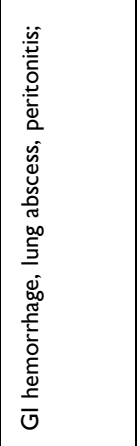 & 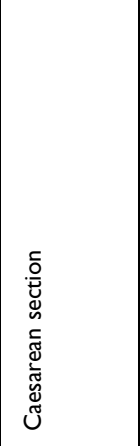 & 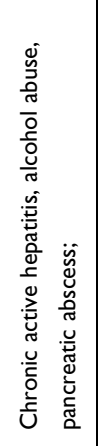 & 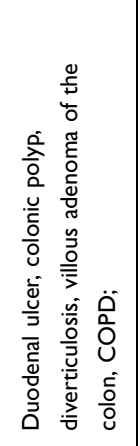 & 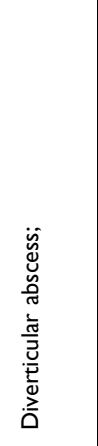 & 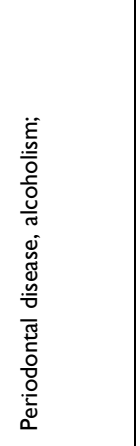 & 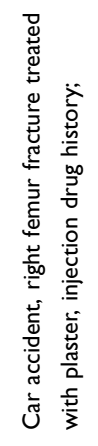 & 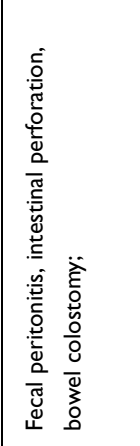 & 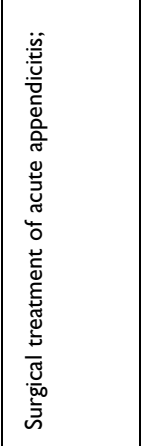 & 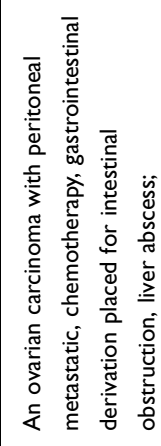 \\
\hline 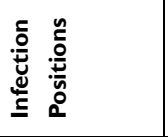 & $\begin{array}{l}\frac{0}{0} \\
\frac{0}{\infty}\end{array}$ & $\begin{array}{l}\frac{0}{0} \\
\frac{\circ}{\infty}\end{array}$ & 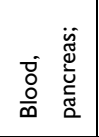 & $\begin{array}{l}\frac{0}{0} \\
\frac{\circ}{\infty}\end{array}$ & 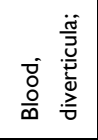 & 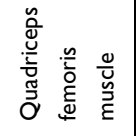 & 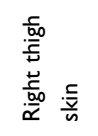 & \begin{tabular}{|l|} 
\\
$\frac{\mathrm{o}}{\infty}$ \\
\end{tabular} & \begin{tabular}{|l|} 
\\
$\frac{0}{\infty}$ \\
$\infty$
\end{tabular} & $\stackrel{\bar{o}}{\stackrel{亠}{I}}$ \\
\hline 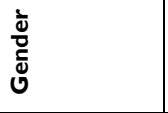 & $\Sigma$ & ч & $\Sigma$ & $\Sigma$ & $\Sigma$ & $\Sigma$ & $\Sigma$ & $\Sigma$ & $\Sigma$ & ч \\
\hline$\stackrel{8}{\stackrel{\circ}{\alpha}}$ & m & $\underline{a}$ & $\stackrel{m}{m}$ & $\Phi_{\infty}^{+}$ & $\pi$ & 产 & q & $\infty$ & m & $\mathcal{f}$ \\
\hline 童 & ک & 吕 & 吕 & 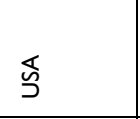 & 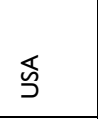 & 竘 & $\frac{\underline{\underline{\underline{T}}}}{\underline{\underline{\underline{J}}}}$ & 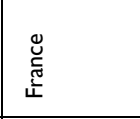 & 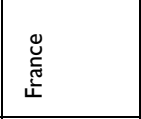 & 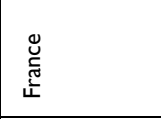 \\
\hline ปัّ & $\stackrel{\infty}{\stackrel{\infty}{a}}$ & $\stackrel{\infty}{\stackrel{\infty}{\sigma}}$ & $\stackrel{\tilde{\varrho}}{\stackrel{0}{\varrho}}$ & $\underset{\stackrel{\infty}{\infty}}{\stackrel{\infty}{\varrho}}$ & 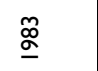 & $\stackrel{\circ}{\circ}$ & ఇ্̊̊ণ & 商 & 商 & 商 \\
\hline
\end{tabular}




\begin{tabular}{|c|c|c|c|c|c|c|c|}
\hline Б्रू & $\Xi$ & $\overline{\bar{n}}$ & हृ & $\overline{\tilde{n}}$ & 彭 & $\Xi$ & 居 \\
\hline 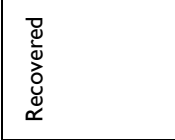 & 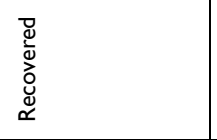 & 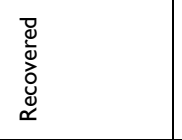 & 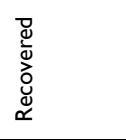 & 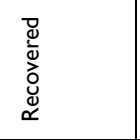 & 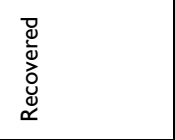 & 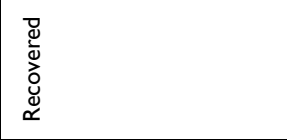 & 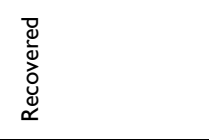 \\
\hline 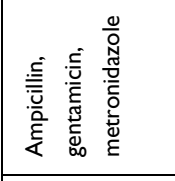 & 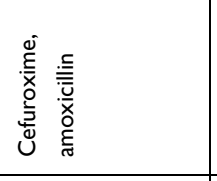 & 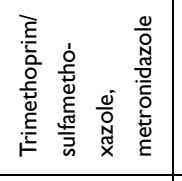 & 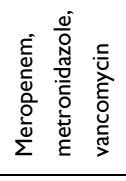 & 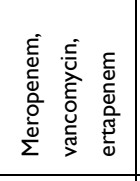 & 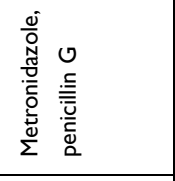 & 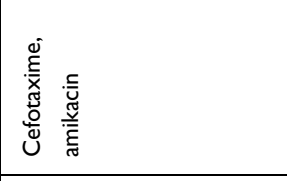 & 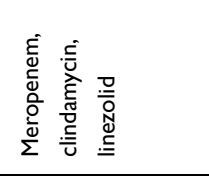 \\
\hline zo & 울 & 울 & 우 & zo & 우 & $\stackrel{\Xi}{\check{\nu}}$ & $\stackrel{\check{\Xi}}{\check{\nu}}$ \\
\hline 1 & $\begin{array}{l}a \\
\text { बे }\end{array}$ & 1 & $\begin{array}{l}\text { 응 } \\
\text { 슴 }\end{array}$ & $\begin{array}{l}\circ \\
\stackrel{\triangle}{\Delta}\end{array}$ & 1 & $\begin{array}{l}\text { n } \\
\text { I̊ } \\
\end{array}$ & 1 \\
\hline 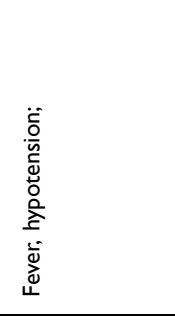 & 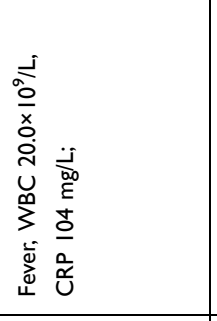 & 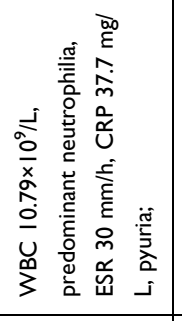 & 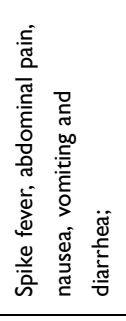 & 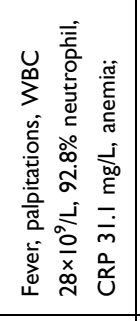 & 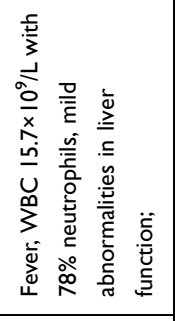 & 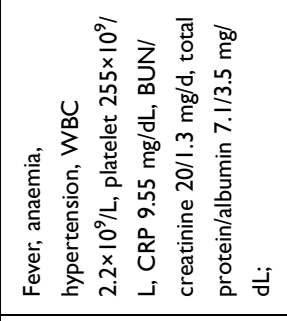 & 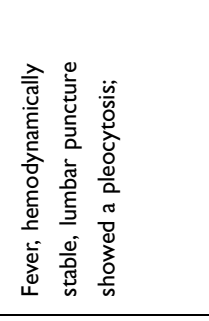 \\
\hline 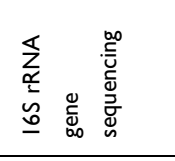 & 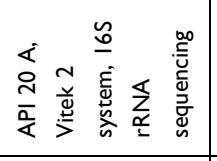 & 1 & 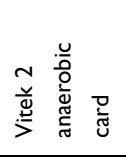 & 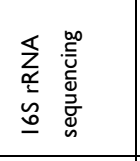 & 1 & 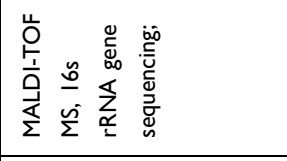 & 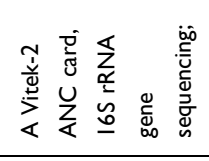 \\
\hline 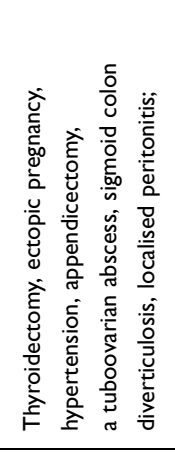 & 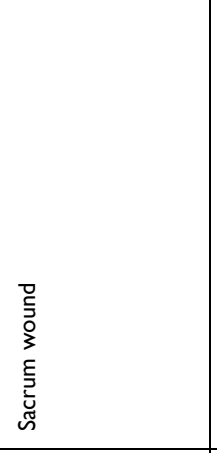 & 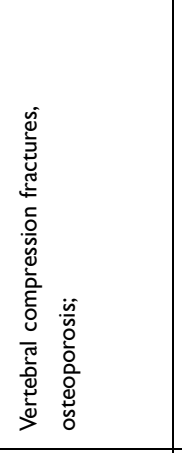 & 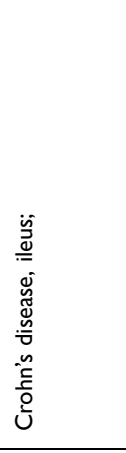 & 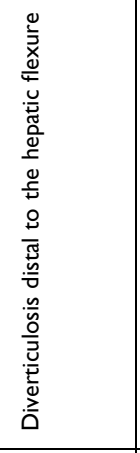 & 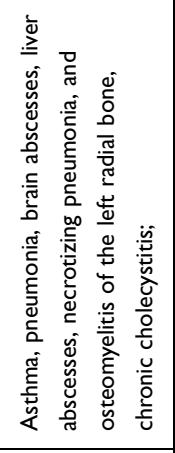 & 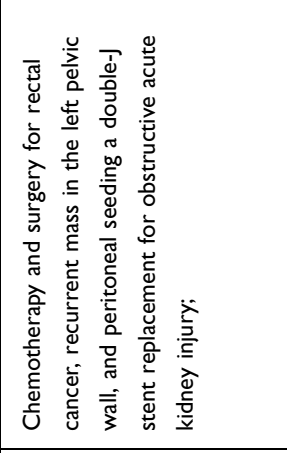 & 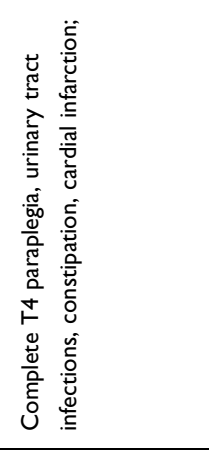 \\
\hline 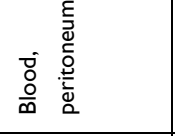 & $\begin{array}{l}\text { o } \\
\text { 음 } \\
\end{array}$ & 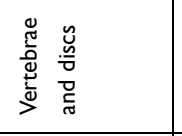 & $\begin{array}{l}\mathrm{o} \\
\frac{0}{\infty} \\
\end{array}$ & $\stackrel{\overline{\bar{D}}}{\stackrel{2}{Z}}$ & $\begin{array}{l}\bar{\delta} \\
\frac{\circ}{\infty}\end{array}$ & \begin{tabular}{|l} 
\\
$\frac{0}{\infty}$ \\
\end{tabular} & $\begin{array}{l}\overline{0} \\
\frac{\circ}{\infty}\end{array}$ \\
\hline 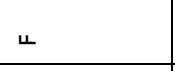 & 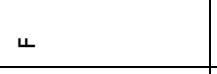 & $\Sigma$ & 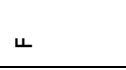 & $\Sigma$ & $\Sigma$ & $\Sigma$ & $\Sigma$ \\
\hline$\stackrel{\infty}{\infty}$ & œ & $\infty$ & $\bar{\sim}$ & $\stackrel{\infty}{\wedge}$ & 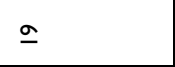 & กิ & R \\
\hline 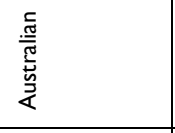 & 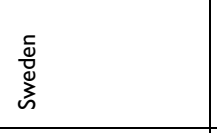 & 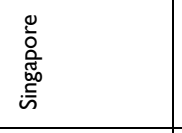 & 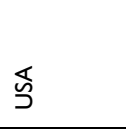 & ऽ & ऽั & 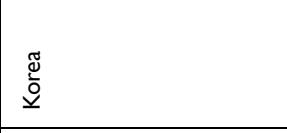 & 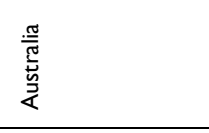 \\
\hline 㐫 & ఫ્రి & ఫ్రి & $\overline{\bar{i}}$ & $\overline{\bar{i}}$ & $\stackrel{\sim}{\bar{i}}$ & $\frac{m}{a}$ & $\stackrel{+}{\grave{a}}$ \\
\hline
\end{tabular}




\begin{tabular}{|c|c|c|c|c|c|c|}
\hline 灾 $\dot{z}$ & 商 & $\stackrel{\Xi}{\varrho}$ & 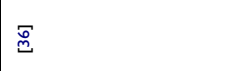 & 菟 & 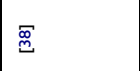 & $\bar{g}$ \\
\hline 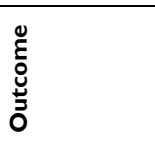 & 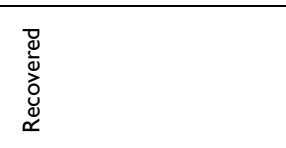 & 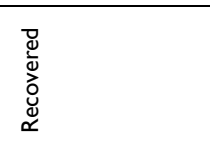 & 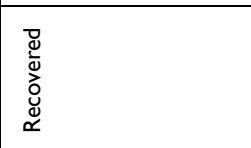 & 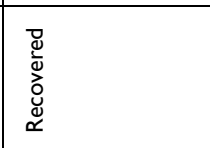 & 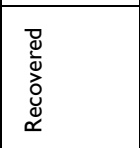 & ס्यू \\
\hline 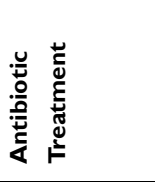 & 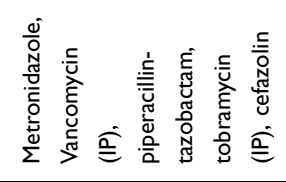 & 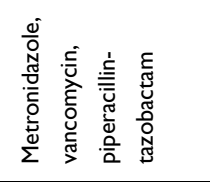 & 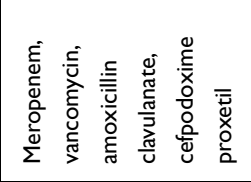 & 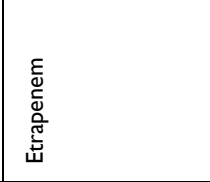 & 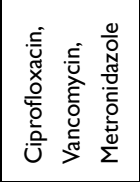 & 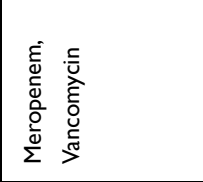 \\
\hline 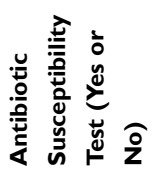 & io & $\stackrel{\check{\nu}}{\tau}$ & $\stackrel{\check{\Delta}}{\check{0}}$ & $\stackrel{\check{\Xi}}{\sim}$ & $\stackrel{\check{g}}{\sim}$ & $\stackrel{\check{\Xi}}{\sim}$ \\
\hline 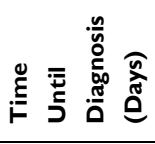 & ๑̊ & $\stackrel{m}{\text { I̊ }}$ & 命 & $\begin{array}{l}\circ \\
\stackrel{̊}{\triangle}\end{array}$ & 승 & 1 \\
\hline 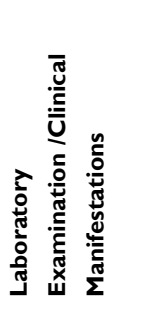 & 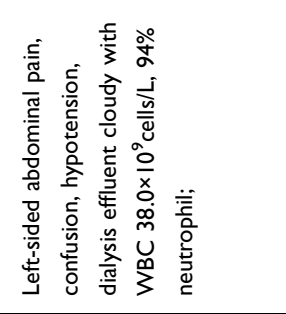 & 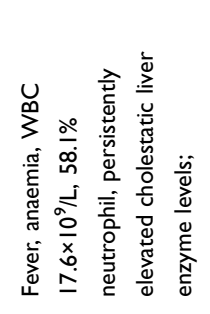 & 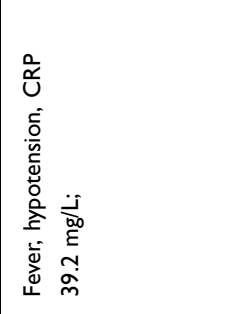 & 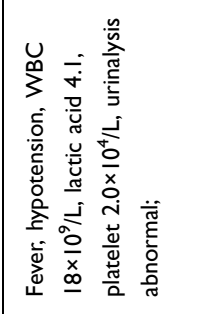 & 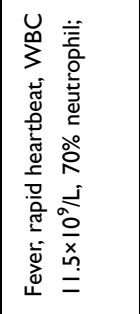 & 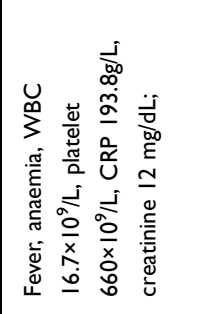 \\
\hline 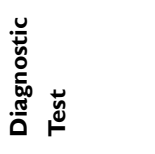 & 1 & 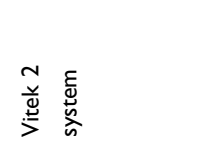 & 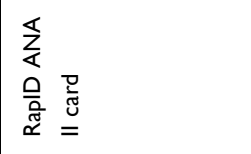 & 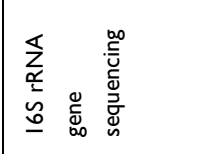 & $\begin{array}{l}\frac{\pi}{\alpha} \\
\frac{\pi}{2} \\
\frac{\pi}{4}\end{array}$ & 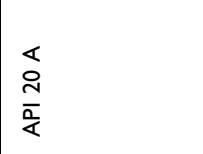 \\
\hline 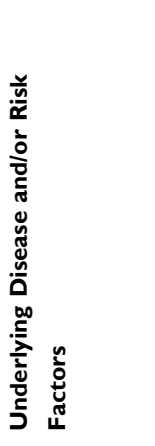 & 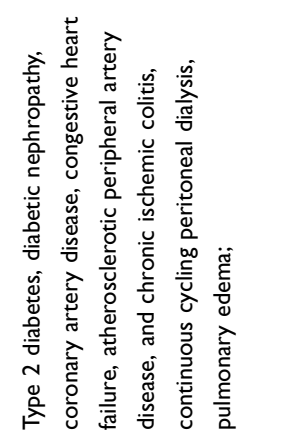 & 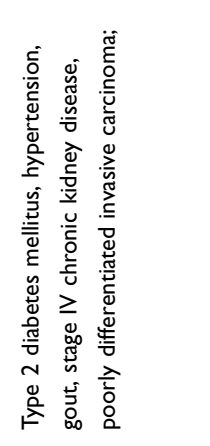 & 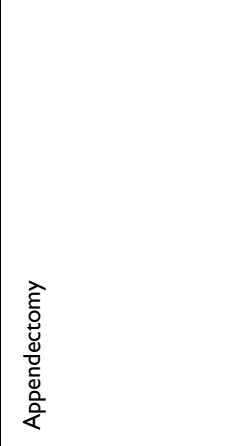 & 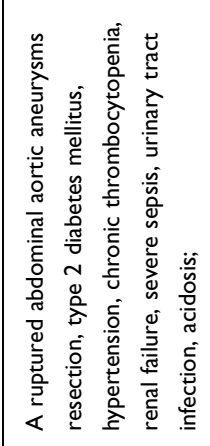 & 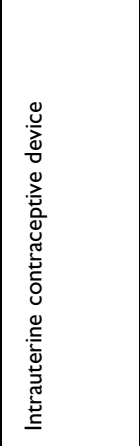 & 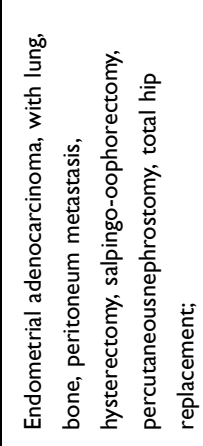 \\
\hline 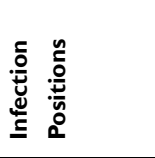 & 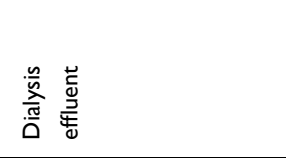 & $\begin{array}{l}\frac{.}{\infty} \\
\frac{0}{\infty}\end{array}$ & 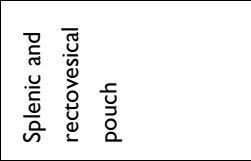 & $\begin{array}{l}\stackrel{ }{0} \\
\frac{\mathrm{o}}{0}\end{array}$ & $\frac{\breve{Z}}{\overline{0}}$ & \begin{tabular}{|l}
$\frac{0}{0}$ \\
$\frac{0}{0}$
\end{tabular} \\
\hline 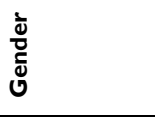 & $\longleftarrow$ & $\Sigma$ & $\Sigma$ & $\Sigma$ & ч & ч \\
\hline$\underset{8}{\stackrel{8}{8}}$ & $\star$ & $\infty$ & $\infty$ & n & f & กิ \\
\hline نे & 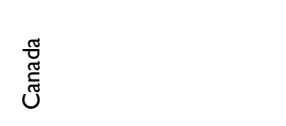 & 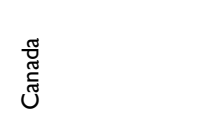 & $\mid \begin{array}{l}\text { 总 } \\
\text { 尊 }\end{array}$ & 䒓 & 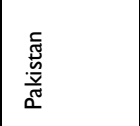 & 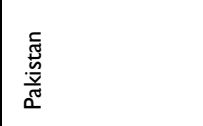 \\
\hline هั & $\frac{\pi}{2}$ & $\frac{t}{\partial}$ & $\bar{n}$ & $\frac{n}{2}$ & $\frac{n}{2}$ & $\hat{\bar{i}}$ \\
\hline
\end{tabular}




\begin{tabular}{|c|c|c|c|c|c|c|}
\hline 至 & 王 & ฐ & $\Xi$ & $\Xi$ & 冞 & 言总 \\
\hline 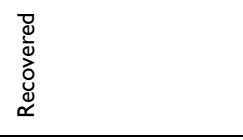 & 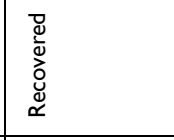 & 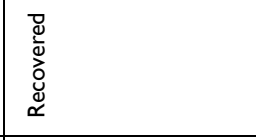 & 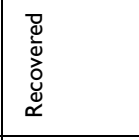 & 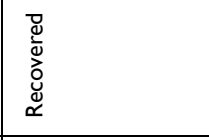 & 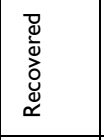 & 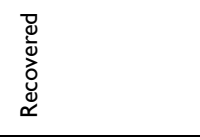 \\
\hline 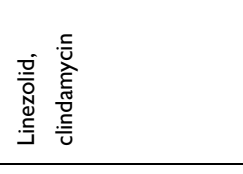 & 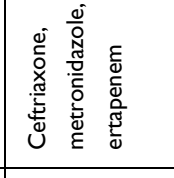 & 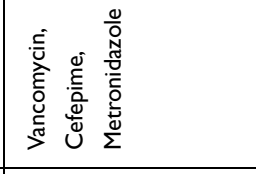 & 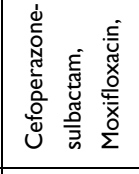 & 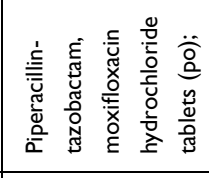 & 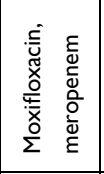 & 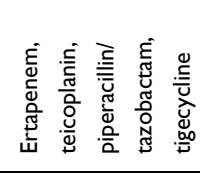 \\
\hline$\stackrel{\mathscr{D}}{0}^{\circ}$ & zo & $\stackrel{0}{z}$ & $\stackrel{0}{z}$ & $\stackrel{0}{z}$ & zo & $\stackrel{\circ}{z}$ \\
\hline 1 & 1 & 1 & 1 & 1 & $\begin{array}{l}+ \\
\text { 今̀ } \\
\end{array}$ & $\begin{array}{l}0 \\
\text { 今̀ } \\
\text { D. }\end{array}$ \\
\hline 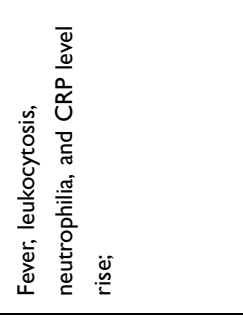 & 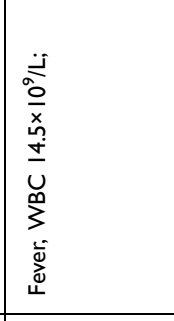 & 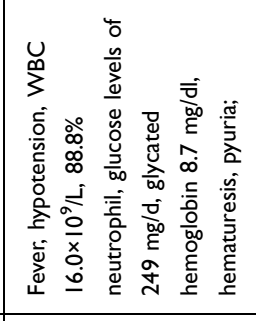 & 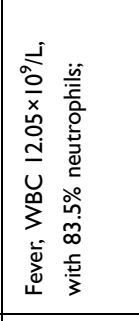 & 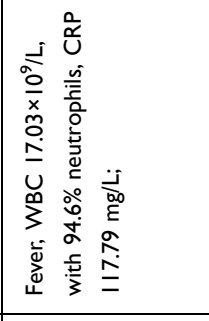 & 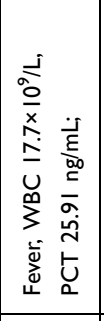 & 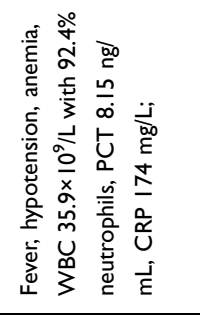 \\
\hline 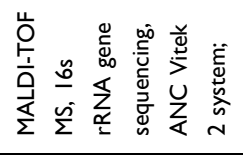 & 1 & 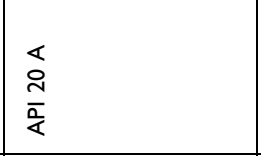 & 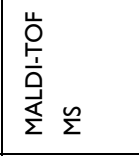 & 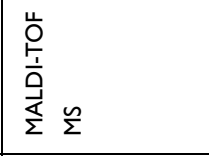 & \begin{tabular}{|l|}
$\frac{0}{0}$ \\
$\frac{1}{0}$ \\
$\frac{1}{\Sigma}$ \\
$\frac{5}{\Sigma}$ \\
\end{tabular} & 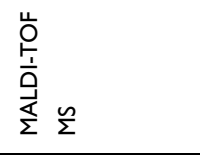 \\
\hline 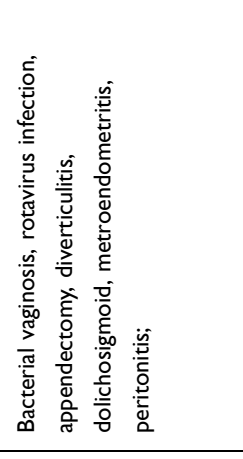 & 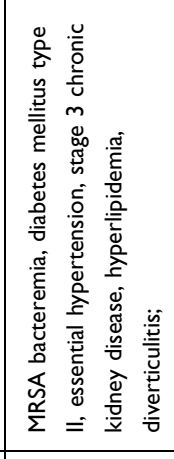 & 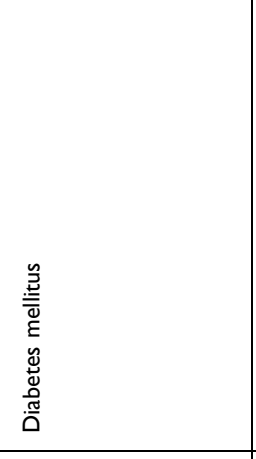 & 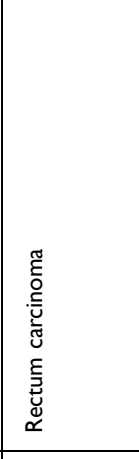 & 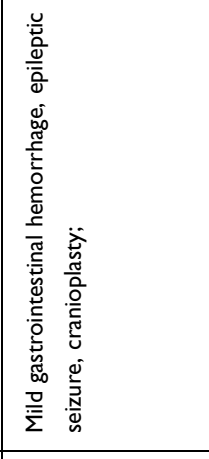 & 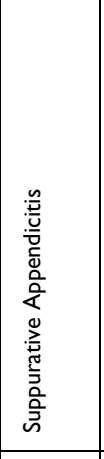 & 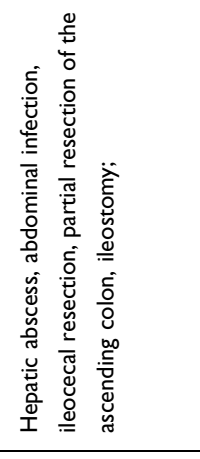 \\
\hline 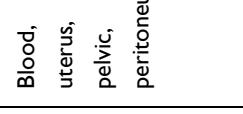 & 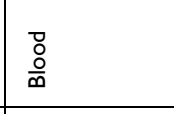 & 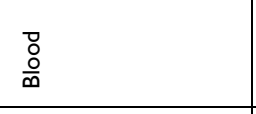 & \begin{tabular}{|l} 
\\
o. \\
$\infty$ \\
\end{tabular} & \begin{tabular}{|l} 
\\
\\
\\
$\infty$
\end{tabular} & \begin{tabular}{|l} 
\\
\\
o. \\
\end{tabular} & $\begin{array}{l}\text { D. } \\
\text { 응 } \\
\end{array}$ \\
\hline$\stackrel{ }{4}$ & $\Sigma$ & $\Sigma$ & $\Sigma$ & $\Sigma$ & $\Sigma$ & $\Sigma$ \\
\hline$\hat{\lambda}$ & J & $\overline{0}$ & t & in & i & $\approx$ \\
\hline 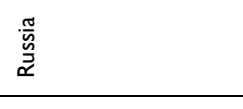 & 乔 & 离 & \begin{tabular}{|l}
$\underline{\underline{E}}$ \\
\end{tabular} & 蚫 & \begin{tabular}{|l}
$\frac{\tilde{E}}{\tilde{\epsilon}}$ \\
\end{tabular} & 营 \\
\hline$\stackrel{\infty}{\stackrel{\infty}{c}}$ & 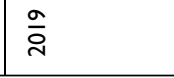 & $\stackrel{\circ}{\bar{c}}$ & ฮี & సี & సี่ & సి \\
\hline
\end{tabular}




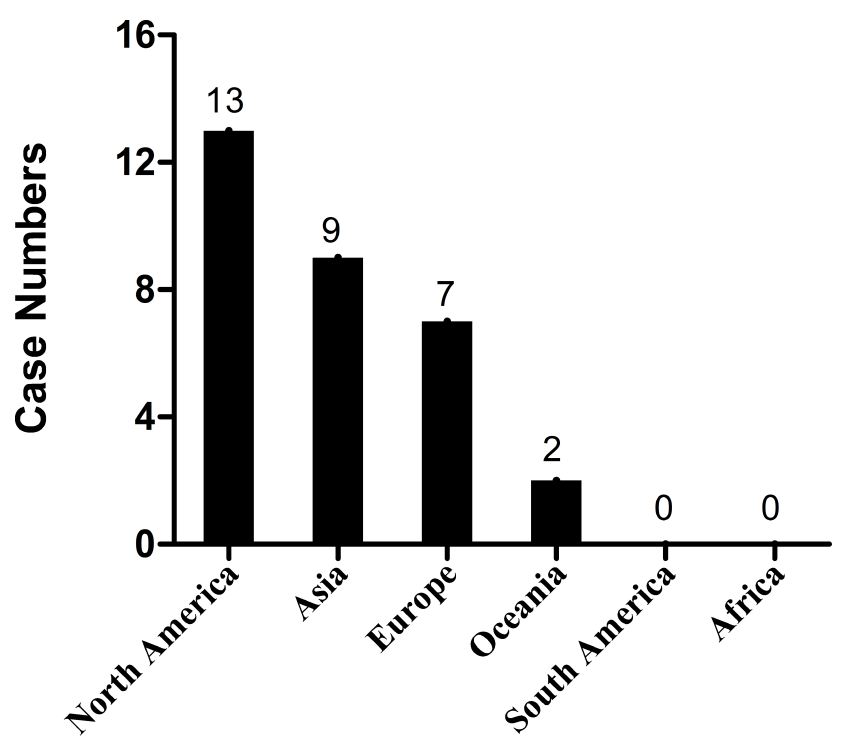

continents

Figure 2 Distribution of sporadic E. lenta infections cases worldwide according to published literature.

sites, $8(25.8 \%)$ cases were in-situ only infections, of which $2(7.1 \%)$ in liver, and 1 each in skin, muscle, pelvis, dialysis effluent, splenic and rectovesical pouch, and vertebrae and discs; 4 cases were in-situ infections documented with blood stream infection, and 19 cases were bloodstream infection without documented origin. The outcomes of all 31 patients were available. Only two $(2 / 31)$ patients died, and the mortality rate was about $6.5 \%$.

\section{Underlying Disease and/or Risk Factors}

The predisposing factors related to the development of E. lenta infection could be grouped into three categories: immunocompromised state (malignancies and diabetes mellitus), potential predisposing condition (surgery, trauma, and bed sores) and digestive system trouble (gastrointestinal pathology and hepatobiliary diseases). All patients had predisposing factors with immunocompromised state in eight $(25.8 \%)$ cases, with potential risk factors in 7 (22.6\%) cases. Fourteen patients (45.2\%) had digestive tract diseases. Two cases had more than one predisposing factor. In fact, the predisposing factors for E. lenta infection in each patient are complex. Almost no one had a single predisposing factor, and the infections were mostly the result of the concurrent effects of various predisposing factors.

\section{Laboratory Examinations and Clinical Manifestations}

Fever was a common presenting symptom, it was recorded in almost all cases, except for two cases who were afebrile and one case whose temperature status was not mentioned. Leukocytosis was reported in $20(64.5 \%)$ cases, and neutrophils were the main cells. The maximum leukocyte counts were up to $38 \times 10^{9} / \mathrm{L}$. Most of the values were between $10 \times 10^{9}$ and $20 \times 10^{9} / \mathrm{L}$ except one patient $\left(2.2 \times 10^{9} / \mathrm{L}\right)$ who underwent chemotherapy for rectal cancer. The PCT and CRP levels measured were all higher than normal. Moreover, the symptoms vary depending on the localization of infection. Many patients also had anemia, hypotension and so on.

\section{Diagnostic Test and Time Until Diagnosis}

Six diagnostic methods were used in the included case reports. Six cases were confirmed by $16 \mathrm{~S}$ rRNA gene sequencing, five infections were identified by API 20 A, four cases were determined by MALDI-TOF MS, two were screened by Minitek System, one was identified by RapID ANA II card, two were confirmed by Vitek-2 ANC card, two cases were determined through the combination of two diagnostic methods, and two cases were found through the combination of three diagnostic methods. Noticeably, no diagnostic test for 7 cases was mentioned.

Time until diagnosis (days) could be determined for 14 patients. The average time of diagnosis was 6.8 days (range 3-10 days), and the median time was 6 days.

\section{AST \& Antibiotic Treatment}

Slightly less than half (14/31) of the patients underwent AST, and no antibiotic susceptibilities were obtained in $54.8 \%(17 / 31)$ of the cases because of the limitations in laboratory conditions. Empirical antibiotic treatment therapy was usually given to the patients who lack AST. The effective treatment regimen mainly focused on combination of carbapenem antibiotics, pencillins, penicillins with inhibitors, cephems antibiotics, nitroimidazole class of antibiotics (metronidazole) and glycopeptide antibiotics.

\section{Discussion}

E. lenta, belonged to the Eggerthellaceae family, is a part of normal human intestinal microbiota. ${ }^{7}$ Due to its fastidious and slow-growing nature in specialized medium, ${ }^{8}$ its culture and identification from clinical specimens is often difficult in microbiology laboratories. Besides, it is often 
overgrown by less fastidious, aerobic organisms. ${ }^{8-10}$ These limitations have delayed the study and understanding of E. lenta. A review of the related literature of 31 sporadic cases of E. lenta infection showed that most of the reported cases came from North America, followed by Asia. Nonetheless, no cases were reported from the South America and Africa (Figure 2), and it is probable that regional differences or under-diagnosis due to lack of technical resources lead to this trend. According to the review, only one case was under 18 years old, most of the patients were middle-aged and elderly, and the average age was 54.3 years. One possible explanation was that older people usually have more comorbidities. The presence of underlying diseases seemed to be a risk factor for E. lenta infection. In our review, the infection rate of E. lenta among males and females was 2.4:1 which indicates that men are more susceptible to it than women. The susceptibility of male to E. lenta infection was assumed to be attributed to the testosterone-mediated immune suppression via their specific receptors while the proinflammatory effect of oestradiol may reduce the incidence of some bacterial infections and associated complications in women. ${ }^{11}$ Until now, the spectrum of diseases caused by this organism has not been well described. To the best of our knowledge, our case is the first report of E. lenta bacteremia related specifically to liver abscess. Although it has also been isolated from various clinical specimens including blood, abscess, appendix, peritoneal fluid, the female genital tract and wound aspirate. ${ }^{12,13}$ E. lenta bacteremia has a high overall mortality rate of $23 \%{ }^{14}$ However, in our review, the total mortality rate of E. lenta clinical infections is only about $6.5 \%$. The most likely explanation was that localized peripheral tissue infection of $E$. lenta aside from bacteremia may bring down the overall mortality rate. The cause of death in one patient with multiple comorbidity conditions (endometrial adenocarcinoma with lung, bone and peritoneum metastases and history of hysterectomy and salpingooophorectomy) was directly attributed to succumb to E. lenta bacteremia.

Except for patients who did not mention relevant information, the body temperature and the leukocyte, PCT and CRP levels of almost all patients were higher than normal, which indicates the presence of infection. However, these clinical manifestations are not unique clinical signs for $E$. lenta infections, but the basic signs for most bacterial infections. An infection that is not initially suspected would increase mortality; ${ }^{15}$ thus, closely monitoring leukocytes, PCT and CRP levels dynamically before identifying the causative microorganism would facilitate treatment as soon as possible and may help reduce the mortality rate. Notably, the body temperature and leukocyte level do not always change synchronously. Venugopal et al suggested that the absence of fever on the day of admission and ICU admission are associated with increased risk of mortality from E. lenta bacteremia, ${ }^{15}$ interestingly, the two patients who were afebrile upon admission were alive. Possibly, E. lenta infection that is confined in peripheral tissue and does not invade the bloodstream has a lower mortality rate than bacteremia. Our patient who was admitted to ICU postoperatively survived possibly because he was given antibiotic therapy even before the identification $E$. lenta growth.

The predisposing factors for E. lenta infection fallen broadly into three categories: immunocompromised state, potential predisposing conditions and digestive system trouble. E. lenta infections are usually associated with risk factors, such as malignancies, diabetes mellitus, surgery, trauma, bed sores, gastrointestinal pathology and hepatobiliary diseases. ${ }^{6,15,16}$ Our study showed that E. lenta infections are mainly related to digestive tract diseases, which is consistent with the results of Alejandra Ugarte-Torres et al. ${ }^{14}$ Three quarters of E. lenta were isolated from blood in our series, including our patient. Although E. lenta is a part of normal human colonic microbiome, ${ }^{17}$ considering its clinical significance when identified in blood cultures, looking for the source and evaluating the patient in detail are advocated. Our patient had liver abscess with perforations in the liver and ascending colon, as well as the resulting abdominal infection, lead to disruption of the intestinal mucosal barrier and raised the possibility of $E$. lenta translocation from the gastrointestinal tract into the bloodstream, it is logical as the source of the patient's infection with E. lenta. Given that the patient's intestinal surgery may have an impact on the continuity of the intestinal mucosa, his bacteremia might have also arisen from the impaired barrier function of intestinal mucosa because of operation.

Since E. lenta was first described in $1935,{ }^{1}$ the techniques used to identify the bacteria have been constantly updated and developed over time. Biochemical typing identification methods, such as Vitek-2 ANC card, API 20A, Minitek System, RapID ANA II card were used initially. Due to its fastidious nature, limitations of conventional phenotypic methods, and lack of effective identification methods, in a lot of center it would be reported as 
"anaerobic Gram positive rod", and many E. lenta infections may have gone undiagnosed previously. With the development of sequencing technology, 16S ribosomal RNA gene sequencing technique was introduced to the clinical microbiologic laboratory. This technique allows the unambiguous identification of pathogens, including E. lenta. However, RNA sequencing is hampered by the quality of the reference sequence database and the difficulty of interpreting the results for most inexperienced users. ${ }^{18}$ More recently, a new technology, matrix-assisted laser desorption ionization-time of flight mass spectrometry (MALDI-TOF MS) was shown to be a valid, fast and accurate identification tool for most clinically relevant anaerobic bacteria. Moreover, a large number of researchers have identified that it is less time-consuming, inexpensive and high throughput; has low sample requirements and does not require specialized personnel. Therefore, this technique has become the new gold standard for the routine identification of clinical anaerobes. ${ }^{19,20}$ In our series, MALDI-TOF MS was used to identify E. lenta since 2013 and has been increasingly widely used over the past few years. The organism in our patient was identified as E. lenta using MALDI-TOF MS, with a score $>2.0$.

E. lenta infections are rare, and its AST is difficult to perform in a microbiology laboratory; therefore, no definitive consensus on the precise antibiotic therapy for this infection was reached. Empiric therapy plays an important role in the treatment of $E$. lenta infections. Only about $40 \%$ of patients were tested for antimicrobial susceptibility in our series. Our patient did not undergo AST because of limitations in laboratory conditions. However, our patient demonstrated good clinical response following the initial empiric treatment with tigecycline and piperacillin/tazobactam, followed by ertapenem and teicoplanin. Bo et al summarised the AST results of $E$. lenta isolates in the last 10 years and showed that isolates remain acceptably susceptible to metronidazole, amoxicillin-clavulanate, carbapenems, vancomycin, cefoxitin, chloramphenicol and clindamycin. ${ }^{21}$ Notably, although all E. lenta isolates were uniformly sensitive to teicoplanin, tigecycline, and carbapenem antibiotics, they exhibit variable susceptibility to piperacillin/tazobactam and moxifloxacin. ${ }^{21}$ Surprisingly, moxifloxacin has good activity against $E$. lenta (minimum inhibitory concentration $[\mathrm{MIC}] \leq 1){ }^{22}$ We speculated that $E$. lenta may acquire resistance to moxifloxacin after multiple steps of mutation over time and geography. Furthermore, the development of reduced sensitivity against piperacillin-tazobactam could be discovered by observing the MIC distributions among the tested E. lenta isolates in some studies. ${ }^{6,23,24}$ One study performed by Ugarte-Torres et al in 2018 demonstrated that empirical monotherapy with piperacillin-tazobactam is associated with increased mortality in patients with $E$. lenta bacteremia. ${ }^{14}$ These data suggest that the clinical efficacy of piperacillin-tazobactam and moxifloxacin are doubtful. Therefore, it is necessary to improve empiric antimicrobial guidelines in order to guide the appropriate management of E. lenta infections. Teicoplanin, vancomycin, amoxicillin-clavulanate, metronidazole, clindamycin, cefoxitin, chloramphenicol, and carbapenems appear to be the most used antibiotic treatment options.

\section{Conclusion}

E. lenta is a rare but significant human neglected pathogen that usually infects elderly patients with various comorbidities especially those with underlying gastrointestinal diseases. Awareness on this uncommon pathogen is increasing with the rapid aging of the population in China and the widespread application of MALDI-TOF MS in modern clinical microbiology laboratories. Laboratory staff and clinicians should pay greater attention to this uncommon bacterium and its clinical importance. Considering that the E. lenta resistance rate has an upward trend around the world, AST should be performed to guide effective antibiotic therapy. Timely and adequate antimicrobial therapy can improve the outcomes of patients with serious E. lenta infections.

\section{Ethics and Consent Statement}

Our study was approved by the Ethics Committee of the First Affiliated Hospital of Shandong First Medical University \& Shandong Provincial Qianfoshan Hospital, and informed consent was obtained from the patient for the publication of this case report and any accompanying images.

\section{Acknowledgment}

We gratefully and genuinely thank Dr. Edward C. Mignot (Shandong University) for linguistic advice.

\section{Author Contributions}

All authors made a significant contribution to the work reported, whether that is in the conception, study design, execution, acquisition of data, analysis and interpretation, or in all these areas; took part in drafting, revising or critically reviewing the article; gave final approval of the version to be published; have agreed on the journal to 
which the article has been submitted; and agree to be accountable for all aspects of the work.

Jiazheng Wang and Rui Guo contributed equally to this work and share the first authorship. Wenyan Xie and Shaofeng Yan contributed equally to this work and share the correspondence authorship.

\section{Funding}

This research was supported by grants from the Shandong Medical and Health Science and Technology Development Plan (2017WS024) and the National Natural Science Foundation of Shandong Province (ZR2019BH062).

\section{Disclosure}

All of the authors declare that the research was conducted in the absence of any commercial or financial relationships. The authors report no conflicts of interest for this work.

\section{References}

1. Eggerth AH. The gram-positive non-spore-bearing anaerobic bacilli of human feces. $J$ Bacteriol. 1935;30(3):277-299. doi:10.1128/ jb.30.3.277-299.1935

2. Kageyama A, Benno Y, Nakase T. Phylogenetic evidence for the transfer of Eubacterium lentum to the genus Eggerthella as Eggerthella lenta gen. nov., comb. nov. Int $J$ Syst Bacteriol. 1999;49(Pt 4):1725-1732.

3. Wade WG, Downes J, Dymock D, et al. The family Coriobacteriaceae: reclassification of Eubacterium exiguum (Poco et al. 1996) and Peptostreptococcus heliotrinreducens (Lanigan 1976) as Slackia exigua gen. nov., comb. nov. and Slackia heliotrinireducens gen. nov., comb. nov., and Eubacterium lentum (Prevot 1938) as Eggerthella lenta gen. nov., comb. nov. Int J Syst Bacteriol. 1999;49(Pt 2):595-600.

4. Saunders E, Pukall R, Abt B, et al. Complete genome sequence of Eggerthella lenta type strain (IPP VPI 0255). Stand Genomic Sci. 2009;1(2):174-182. doi:10.4056/sigs.33592

5. Lee HJ, Hong SK, Choi WS, Kim EC. The first case of Eggerthella lenta bacteremia in Korea. Ann Lab Med. 2014;34(2):177-179. doi:10.3343/alm.2014.34.2.177

6. Lee MR, Huang YT, Liao CH, et al. Clinical and microbiological characteristics of bacteremia caused by Eggerthella, Paraeggerthella, and Eubacterium species at a university hospital in Taiwan from 2001 to 2010 . J Clin Microbiol. 2012;50(6):2053-2055. doi:10.1128/JCM.00548-12

7. Clavel T, Charrier C, Braune A, Wenning M, Blaut M, Haller D. Isolation of bacteria from the ileal mucosa of TNFdeltaARE mice and description of Enterorhabdus mucosicola gen. nov., sp. nov. Int $J$ Syst Evol Microbiol. 2009;59(Pt 7):1805-1812. doi:10.1099/ijs.0.003087-0

8. Lau SK, Woo PC, Fung AM, Chan KM, Woo GK, Yuen KY. Anaerobic, non-sporulating, gram-positive bacilli bacteraemia characterized by $16 \mathrm{~S}$ rRNA gene sequencing. $J$ Med Microbiol. 2004;53(Pt 12):1247-1253. doi:10.1099/jmm.0.45803-0

9. Liderot K, Larsson M, Boräng S, Ozenci V. Polymicrobial bloodstream infection with Eggerthella lenta and Desulfovibrio desulfuricans. J Clin Microbiol. 2010;48(10):3810-3812. doi:10.1128/JCM.02481-09

10. Warren YA, Citron DM, Merriam CV, Goldstein EJ. Biochemical differentiation and comparison of Desulfovibrio species and other phenotypically similar genera. $J$ Clin Microbiol. 2005;43 (8):4041-4045.
11. Vázquez-Martínez ER, García-Gómez E, Camacho-Arroyo I, González-Pedrajo B. Sexual dimorphism in bacterial infections. Biol Sex Differ. 2018;9(1):27.

12. Mosca A, Summanen P, Finegold SM, De Michele G, Miragliotta G. Cellular fatty acid composition, soluble-protein profile, and antimicrobial resistance pattern of Eubacterium lentum. J Clin Microbiol. 1998;36(3):752-755. doi:10.1128/JCM.36.3.752-755.1998

13. Lau SK, Woo PC, Woo GK, et al. Eggerthella hongkongensis sp. nov. and eggerthella sinensis sp. nov., two novel Eggerthella species, account for half of the cases of Eggerthella bacteremia. Diagn Microbiol Infect Dis. 2004;49(4):255-263. doi:10.1016/j. diagmicrobio.2004.04.012

14. Ugarte-Torres A, Gillrie MR, Griener TP, Church DL. Eggerthella lenta bloodstream infections are associated with increased mortality following empiric piperacillin-Tazobactam (TZP) monotherapy: a Population-based Cohort Study. Clin Infect Dis. 2018;67 (2):221-228. doi:10.1093/cid/ciy057

15. Venugopal AA, Szpunar S, Johnson LB. Risk and prognostic factors among patients with bacteremia due to Eggerthella lenta. Anaerobe. 2012;18(4):475-478. doi:10.1016/j.anaerobe.2012.05.005

16. Wong D, Aoki F, Rubinstein E. Bacteremia caused by Eggerthella lenta in an elderly man with a gastrointestinal malignancy: a case report. Can J Infect Dis Med Microbiol. 2014;25(5):e85-e86.

17. Li F, Hullar MA, Schwarz Y, Lampe JW. Human gut bacterial communities are altered by addition of cruciferous vegetables to a controlled fruit- and vegetable-free diet. J Nutr. 2009;139 (9):1685-1691. doi:10.3945/jn.109.108191

18. Woo PC, Lau SK, Teng JL, Tse H, Yuen KY. Then and now: use of 16S rDNA gene sequencing for bacterial identification and discovery of novel bacteria in clinical microbiology laboratories. Clin Microbiol Infect. 2008;14(10):908-934.

19. Patel R. MALDI-TOF MS for the diagnosis of infectious diseases. Clin Chem. 2015;61(1):100-111. doi:10.1373/clinchem.2014.221770

20. Coltella L, Mancinelli L, Onori M, et al. Advancement in the routine identification of anaerobic bacteria by MALDI-TOF mass spectrometry. Eur J Clin Microbiol Infect Dis. 2013;32 (9):1183-1192. doi:10.1007/s10096-013-1865-1

21. Bo J, Wang S, Bi Y, Ma S, Wang M, Du Z. Eggerthella lenta bloodstream infections: two cases and review of the literature. Future Microbiol. 2020;15(11):981-985. doi:10.2217/fmb-2019-0338

22. Liebetrau A, Rodloff AC, Behra-Miellet J, Dubreuil L. In vitro activities of a new des-fluoro(6) quinolone, garenoxacin, against clinical anaerobic bacteria. Antimicrob Agents Chemother. 2003;47 (11):3667-3671. doi:10.1128/AAC.47.11.3667-3671.2003

23. Gardiner BJ, Tai AY, Kotsanas D, et al. Clinical and microbiological characteristics of Eggerthella lenta bacteremia. J Clin Microbiol. 2015;53(2):626-635. doi:10.1128/JCM.02926-14

24. Nagaoka R, Kitagawa H, Koba Y, et al. Clinical and microbiological characteristics of Eggerthella lenta bacteremia at a Japanese tertiary hospital. J Infect Chemother. 2021;27(8):1261-1264. doi:10.1016/j. jiac.2021.03.019

25. Bourne KA, Beebe JL, Lue YA, Ellner PD. Bacteremia due to Bifidobacterium, Eubacterium or Lactobacillus; twenty-one cases and review of the literature. Yale J Biol Med. 1978;51(5):505-512.

26. Eng RH, Suwanagool S, Chmel H, Smith SM, Tecson-Tumang F, Corrado M. The significance of eubacterium bacteremia. Am J Gastroenterol. 1983;78(2):90-93.

27. Palomino-Nicás J, González E, Arroyo A, Cañas E, Hernanz W, Pachón J. Pyomyositis due to Eubacterium lentum and Streptococcus constellatus from a periodontal source. Clin Infect Dis. 1996;22(1):176-178. doi:10.1093/clinids/22.1.176

28. Lattuada E, Zorzi A, Lanzafame M, et al. Cutaneous abscess due to Eubacterium lentum in injection drug user: a case report and review of the literature. $J$ Infect. 2005;51(2):E71-E72. doi:10.1016/j. jinf.2004.08.026 
29. Landais C, Doudier B, Imbert G, Fenollar F, Brouqui P. Application of rrs gene sequencing to elucidate the clinical significance of Eggerthela lenta infection. J Clin Microbiol. 2007;45(3):1063-1065. doi:10.1128/JCM.01805-06

30. Chan RC, Mercer J. First Australian description of Eggerthella lenta bacteraemia identified by $16 \mathrm{~S}$ rRNA gene sequencing. Pathology. 2008;40(4):409-410. doi:10.1080/00313020802036772

31. Bok CW, Ng YS. Eggerthella lenta as a cause of anaerobic spondylodiscitis. Singapore Med J. 2009;50(12):e393-e396.

32. Thota VR, Dacha S, Natarajan A, Nerad J. Eggerthella lenta bacteremia in a crohn's disease patient after ileocecal resection. Future Microbiol. 2011;6(5):595-597. doi:10.2217/fmb.11.31

33. Salameh A, Klotz SA, Zangeneh TT. Disseminated infection caused by eggerthella lenta in a previously healthy young man: a case report. Case Rep Infect Dis. 2012;2012:517637. doi:10.1155/2012/517637

34. Gardiner BJ, Korman TM, Junckerstorff RK. Eggerthella lenta bacteremia complicated by spondylodiscitis, psoas abscess, and meningitis. J Clin Microbiol. 2014;52(4):1278-1280. doi:10.1128/ JCM.03158-13

35. Goupil R, Nadeau-Fredette AC, Tennankore KK, Bargman JM. Peritonitis caused by veillonella species and Eggerthella lenta in peritoneal dialysis. Perit Dial Int. 2014;34(2):245-247. doi:10.3747/ pdi.2013.00046

36. Kondo S, Okada H, Shimono R, Kusaka T. Paediatric splenic and rectovesical pouch abscesses caused by Eggerthella lenta. BMJ Case Rep. 2015;2015(jun03 1):bcr2015209584. doi:10.1136/bcr-2015209584
37. Jehangir W, Sanabria F, Singh Z, et al. Enteric fistulous communication with an aortobifemoral graft permitting for Eggerthella Lenta colonization. J Glob Infect Dis. 2015;7(4):170-172. doi:10.4103/ 0974-777X.170506

38. Mal PB, Rafiq I, Iftikhar I, Irfan S. Pelvic abscess caused by a slow growing anaerobic bacterium, Eggerthella lenta: first case report from Pakistan. J Pak Med Assoc. 2017;67(10):1604-1605.

39. Fatima T, Hasan R, Tariq S. Eggerthella lenta bacteraemia in endometrial adenocarcinoma - a case report from Pakistan. J Pak Med Assoc. 2020;70(6):1079-1080.

40. Priputnevich T, Lyubasovskaya L, Muravieva V, et al. Postpartum endometritis and obstetrical sepsis associated with Eggerthella lenta. Case report and review of the literature. J Matern Fetal Neonatal Med. 2021;34(2):313-317. doi:10.1080/14767058.2019.1602602

41. Peter-Bibb TK, Tokeshi J. Hawaii's first published case of Eggerthella lenta sepsis. Hawaii J Health Soc Welf. 2020;79 (11):326-328.

42. Cordoba G, Kim ML, Sharma S, Paniagua J, Folgarait G, Berger J. Septic shock caused by the under-recognized bacterium Eggerthella lenta in a 61-year-old male with a periurethral abscess: a case report. Rev Soc Bras Med Trop. 2019;52:e20190081. doi:10.1590/00378682-0081-2019

43. Jiang J, She B, Zheng R. Bacteremia caused by the Eggerthella lenta in a previously healthy 30 -year-old man with acute suppurative appendicitis: a case report from China. Infect Drug Resist. 2020;13:3695-3698. doi:10.2147/IDR.S274494
Infection and Drug Resistance

\section{Publish your work in this journal}

Infection and Drug Resistance is an international, peer-reviewed openaccess journal that focuses on the optimal treatment of infection (bacterial, fungal and viral) and the development and institution of preventive strategies to minimize the development and spread of resistance. The journal is specifically concerned with the epidemiology of antibiotic resistance and the mechanisms of resistance development and diffusion in both hospitals and the community. The manuscript management system is completely online and includes a very quick and fair peerreview system, which is all easy to use. Visit http://www.dovepress.com/ testimonials.php to read real quotes from published authors. 